\title{
Gravitational loss-cone instability in stellar clusters around massive black holes
}

\author{
Evgeny V. Polyachenko ${ }^{1}$, Valerij L. Polyachenko ${ }^{1}$ and \\ Ilia G. Shukhman ${ }^{2}$ \\ ${ }^{1}$ Institute of Astronomy RAS, Moscow, Russia \\ email: epolyach@inasan.ru \\ ${ }^{2}$ Institute of Solar-Terrestrial Physics, RAS SB, Irkutsk, Russia \\ email: shukhman@iszf.irk.ru
}

\begin{abstract}
Stability of spherical and thin disk stellar clusters surrounding massive black holes are studied. Due to the black hole, stars with sufficiently low angular momenta escape from the system through the loss cone. We show that stability of spherical clusters crucially depend on whether the distribution of stars is monotonic or non-monotonic in angular momentum. It turns out that only non-monotonic distributions can be unstable. At the same time the instability in disk clusters is possible for both types of distributions.
\end{abstract}

Keywords. Galaxy: center, galaxies: nuclei

\section{Introduction}

Study of the gravitational loss-cone instability, an analog of the plasma cone instability, has begun from Polyachenko (1991) work in which a simplest analytical model of thin disk stellar cluster has been employed. The interest to the problem of stability of stellar clusters has been revived recently by detailed investigation by Tremaine (2005) and Polyachenko, Polyachenko \& Shukhman (2007; henceforth Paper I) of low mass clusters around massive black holes. The both papers have considered stability of small amplitude perturbations of stellar clusters of disk-like and spherical geometry.

Tremaine (2005) has shown that thin disk with a symmetrical DF possessing an empty loss cone is generally unstable. On the contrary, analyzing perturbations with spherical numbers $l=1$ and $l=2$, he deduced that spherical clusters with DF monotonically growing in angular momentum should be generally stable.

In Paper I, however, we have demonstrated that spherical systems with non-monotonic distributions may be unstable for sufficiently small-scale perturbations $l \geqslant 3$, while the harmonics $l=1,2$ remain stable as well. For the sake of convenience, we have used two assumptions: i) Keplerian potential of the massive black hole dominates over selfgravitating potential of the stellar cluster; ii) the stellar system is populated mainly by near-radial orbits (a so called spoke approximation). Then the evolution of the system can be traced on slow precessing timescales, rather than typical free fall timescales. The spoke approximation reduces the problem to study of rather simple analytical characteristic equations for small perturbations of stellar clusters.

There are two questions that naturally arise in this context: i) Does the instability remain if we go beyond the spoke approximation? ii) Does the instability occur in spheres with monotonically increasing distributions in angular momentum if one consider smallerscale perturbations with $l \geqslant 3$ ?

To achieve the task we use semi-analytical approach based on analysis of integral equations for slow modes elaborated recently in Polyachenko $(2004,2005)$ for thin disks, 
and in Paper I for spherical geometry. Following Paper I, we restrict ourselves to studying monoenergetic models with DFs in the form $F(E, L)=A \delta\left(E-E_{0}\right) f(L)$. Models are specified by functions $f(L)$.

\section{Variational principle and zero mode}

From integral equations (Paper I) for spheres and for thin disks one can conclude that i) if DF is monotonic, then only aperiodic solutions are possible, and ii) periodic (rotating) solutions are possible in case of non-monotonic DF.

If $\mathrm{DF}$ is monotonic, terms in the equations can be divided according to their contribution to instability, and a simple mechanical interpretation with positive and negative elastic forces is possible. Then a so called variation principle takes place. If exists, the instability would be of hydrodynamic type (like Jeans or radial orbit instabilities), when all particles are involved. On the other hand, periodic (rotating) solutions are possible in case of non-monotonic DF only. The mechanical interpretation does not work in this case. Then, the instability has a kinetic nature, in which small amount of resonance particles are responsible for the mode growth.

Tremaine (2005) has shown that for an arbitrary spherical DF $F(E, L)$ with empty loss cone, $F(E, L=0)=0$, a zero frequency lopsided mode $l=1$ must exist. The mode corresponds to a non-trivial perturbation arising under shift of the spherical system as a whole relative to the central mass.

\section{Results}

Existence of the zero mode for spherical $l=1$ perturbations brings serious doubts that models with monotonous DFs $f(L)$ can be unstable. Indeed, the role of destabilizing contribution in the integral equation falls off with increasing the number $l$. So, it is expected that the most dangerous modes correspond to the lowest values of $l$. But it turns out from numerical analysis that $l=1$ and $l=2$ cases are stable, as well as smaller scale mode $l \geqslant 3$.

On the other hand, non-monotonic DFs provide examples of gravitational loss-cone instability, which occurs at spherical harmonics $l \geqslant 3$ and remains effective for systems with non-radial orbits.

Unlike spheres, disk-like systems are usually unstable. It can be proved that disks with symmetrical DFs and $d f / d|L|>0$ with empty loss cones are unstable with respect to perturbations with azimuthal number $m=1$.

The work was supported by Russian Science Support Foundation, RFBR grants No. 0502-17874 and No. 07-02-00931, "Leading Scientific Schools" Grant No. 7629.2006.2 and "Young doctorate" Grant No. 2010.2007.2 provided by the Ministry of Industry, Science, and Technology of Russian Federation, and the "Extensive objects in the Universe" Grant provided by the Russian Academy of Sciences, and also by Programs of presidium of Russian Academy of Sciences No 16 and OFN RAS No 16.

\section{References}

Polyachenko V. L. 1991, Soviet Astron. Lett., 17, 371

Polyachenko E. V. 2004, MNRAS, 348, 345

Polyachenko E. V. 2005, MNRAS, 357, 559

Polyachenko E. L., Polyachenko V. L., \& Shukhman I. G. 2007, MNRAS, 379, 573

Tremaine S., 2005, ApJ, 625, 143 